\title{
WANDERING DOMAINS IN QUASIREGULAR DYNAMICS
}

\author{
DANIEL A. NICKS
}

(Communicated by Mario Bonk)

\begin{abstract}
We show that wandering domains can exist in the Fatou set of a polynomial type quasiregular mapping of the plane. We also give an example of a quasiregular mapping of the plane, with an essential singularity at infinity, which has a sequence of wandering domains contained in a bounded part of the plane. This contrasts with the situation in the analytic case, where wandering domains are impossible for polynomials and, for transcendental entire functions, the existence of wandering domains in a bounded part of the plane has been an open problem for many years.
\end{abstract}

\section{INTRODUCTION}

One generalisation of nonlinear polynomials in the complex plane is the family of quasiregular mappings of polynomial type for which the degree exceeds the dilatation. The definition of these terms, and a number of others, is deferred to the next section. Here we mention only that a quasiregular map $f: \mathbb{R}^{m} \rightarrow \mathbb{R}^{m}$ is said to be of polynomial type if $f(x) \rightarrow \infty$ as $x \rightarrow \infty$ and that the degree of such a mapping is defined as the maximal number of preimages of any value.

Suppose that an open set $U$ is completely invariant under a mapping $f$. Let $U_{0}$ be a component of $U$ and let $U_{n}$ denote that component of $U$ which contains $f^{n}\left(U_{0}\right)$. Then we say that $U_{0}$ is a wandering component or a wandering domain of $U$ if $U_{n} \neq U_{m}$ whenever $n \neq m$.

Motivated by Sullivan's celebrated proof that the Fatou set of a rational function has no wandering components [10], Lasse Rempe asked the following question.

Question ([14, Problem 6, pp. 2959-2960]). Let $p: \mathbb{R}^{2} \rightarrow \mathbb{R}^{2}$ be a $K$-quasiregular mapping of polynomial type, $\operatorname{such}$ that $\operatorname{deg} p>K$. Can the Fatou set of $p$ have a wandering component?

Before tackling this question, we must consider what we mean by the Fatou and Julia sets of a quasiregular mapping. For a polynomial, it is well known that the Julia set is the boundary of the escaping set. We have the following result about the escaping sets of quasiregular mappings of polynomial type. Here, the winding map $r e^{i \theta} \mapsto r e^{2 i \theta}$ shows the sharpness of the degree condition.

Theorem $1.1([5])$. Let $p: \mathbb{R}^{m} \rightarrow \mathbb{R}^{m}$ be a $K$-quasiregular mapping of polynomial type, such that $\operatorname{deg} p>K$. Then the escaping set

$$
I(p)=\left\{x \in \mathbb{R}^{m}: p^{n}(x) \rightarrow \infty \text { as } n \rightarrow \infty\right\}
$$

Received by the editors January 7, 2011 and, in revised form, August 23, 2011.

2010 Mathematics Subject Classification. Primary 30C62, 30C65, 37F50; Secondary 37F10. 
is nonempty, open and connected. The boundary $\partial I(p)$ is a perfect set and is completely invariant under $p$.

This result suggests that the boundary of the escaping set $\partial I(p)$ may serve as an analogue to the Julia set of a polynomial. In light of this, we could attempt to answer Rempe's question by seeking wandering components of $\overline{I(p)} c=\mathbb{R}^{2} \backslash \overline{I(p)}$.

An alternative interpretation of the above question is offered by the work of Sun and Yang [11, 12, 13] (see also [2]), which considers quasiregular mappings of the two-dimensional Riemann sphere. They define the Julia set $J(p)$ of a polynomial type quasiregular mapping $p: \mathbb{R}^{2} \rightarrow \mathbb{R}^{2}$ as the set of points $x \in \mathbb{R}^{2}$ with the following expanding property: if $U$ is any neighbourhood of $x$, then $\bigcup_{n} p^{n}(U)$ contains all but at most one point of $\mathbb{R}^{2}$. Sun and Yang prove that if $\operatorname{deg} p>K(p)$, then the Julia set is nonempty, perfect and completely invariant. It is easy to see that $J(p) \subseteq \partial I(p)$.

For polynomial type mappings $p$ as above, Sun and Yang retain the familiar definition of the Fatou set: $x \in F(p)$ if there exists a neighbourhood $U$ of $x$ such that $\left\{\left.p^{n}\right|_{U}\right\}$ is a normal family. It should be remarked that under these definitions $\mathbb{R}^{2} \backslash(J(p) \cup F(p))$ can be nonempty; see, for example, [2, Example 5.3].

This note answers Rempe's question by constructing a suitable mapping $p$ that has wandering domains. Our approach is based on the escaping set.

Theorem 1.2. There exists a quasiregular map $p: \mathbb{R}^{2} \rightarrow \mathbb{R}^{2}$ of polynomial type, with $\operatorname{deg} p>K(p)$, for which there are wandering components of both $\overline{I(p)}^{c}$ and of the Fatou set $F(p)$.

The construction described in Sections 3.1 and 3.2 will establish Theorem 1.2 . A further interesting feature of this example is that the Julia set is not equal to the boundary of the escaping set, in contrast to the polynomial case. In [2, Example 5.3], it was already shown that for a polynomial type mapping with $\operatorname{deg} p>K(p)$, the boundary of an attracting basin may not coincide with the Julia set. Note, however, that in our case the attracting fixed point at infinity is even superattracting.

In Section 3.3, we adapt the construction to produce a polynomial type map of any degree $d \geq 2$, with dilatation arbitrarily close to 1 and which possesses wandering Fatou components.

In the final section, we modify one of our functions to create a 'transcendental' quasiregular mapping of the plane that exhibits wandering in a bounded region. That is, we describe a quasiregular map, with an essential singularity at infinity, which has a wandering domain on which the iterates converge to a finite constant.

\section{Definitions}

Let $G \subseteq \mathbb{R}^{2}$ be a domain. A continuous function $f: G \rightarrow \mathbb{R}^{2}$ is called $K$-quasiregular if it belongs to the Sobolev space $W_{2, \text { loc }}^{1}(G)$ and

$$
|D f(x)|^{2} \leq K J_{f}(x),
$$

for almost every $x \in G$. Here $J_{f}(x)$ denotes the Jacobian determinant of $f$ at the point $x$, while $|D f(x)|=\sup _{|a|=1}|D f(x) a|$ is the operator norm of the formal derivative of $f$ at $x$. The least $K \geq 1$ for which this holds is called the dilatation $K(f)$. A function is said to be quasiregular if it is $K$-quasiregular for some $K$. 
Any analytic function is 1-quasiregular, and indeed quasiregular mappings are a natural generalisation of analytic functions. The definition of $K$-quasiregularity in higher dimensions differs slightly from the above and may be found in [2, 9], along with more details of many of the concepts defined here.

A nonconstant quasiregular mapping $f: \mathbb{R}^{2} \rightarrow \mathbb{R}^{2}$ is of polynomial type if $f(x) \rightarrow \infty$ as $x \rightarrow \infty$. Otherwise, this limit does not exist and $f$ is said to have an essential singularity at infinity. The degree of $f$ can be defined by

$$
\operatorname{deg} f:=\sup _{y \in \mathbb{R}^{2}}\left|f^{-1}(y)\right|
$$

that is, the maximal number of preimages of any value in $\mathbb{R}^{2}$. It is well known that $\operatorname{deg} f$ is finite if and only if $f$ is of polynomial type.

An injective quasiregular mapping is called quasiconformal. Any quasiregular map $f: \mathbb{R}^{2} \rightarrow \mathbb{R}^{2}$ has a Stoilow factorisation of the form $f=g \circ h$, where $h$ is quasiconformal and $g$ is analytic [2, §3.2]. Note that if $f$ is of polynomial type, then $g$ is a polynomial.

The composition of two quasiregular maps $f_{1}$ and $f_{2}$ is quasiregular with dilatation satisfying $K\left(f_{1} \circ f_{2}\right) \leq K\left(f_{1}\right) K\left(f_{2}\right)$. In particular, the iterates of a quasiregular mapping are also quasiregular.

\section{The MAIN CONSTRUCTION}

We answer the question discussed in the introduction by describing a polynomial type quasiregular mapping that has wandering domains.

3.1. Two quasiconformal maps. The construction takes place in a number of stages, and we shall identify $\mathbb{R}^{2}$ with $\mathbb{C}$. To begin, let $W_{0}$ be the open diamond with vertices at $i, \frac{i}{2},(3 i+1) / 4$ and $(3 i-1) / 4$. See Figure 1 . For $y \in\left[\frac{1}{2}, 1\right]$, we define a continuous function $h_{y}: \mathbb{R} \rightarrow \mathbb{R}$ by setting $h_{y}(x)=1$ when $x+i y \in W_{0}$, while $h_{y}(x)=4$ when $|x| \geq y$. For intermediate values of $x$, we ask that $h_{y}$ be linear in $x$. Explicitly, in the regions where $h_{y}$ is not locally constant this gives

$$
h_{y}(x)= \begin{cases}6|x|-6 y+4, & \frac{1}{2} \leq y \leq \frac{3}{4}, \\ \frac{3|x|+5 y-4}{2 y-1}, & \frac{3}{4}<y \leq 1 .\end{cases}
$$

Write $S=\left\{z: \frac{1}{2}<\operatorname{Im} z<1\right\}$. We now define a homeomorphism $h: \bar{S} \rightarrow \bar{S}$ by

$$
h(x+i y)=h_{y}(x) x+i y,
$$

so that $h$ is locally like a stretch by a factor $h_{y}(x)$ parallel to the real axis. We shall later use the fact that

$$
h(x+i / 2)=h(2 x+i) / 2 .
$$

We show next that $h$ is quasiconformal on $S$. Note that $h$ is differentiable almost everywhere in $S$ and that $\operatorname{Im}(h(x+i y))$ is independent of $x$. Hence, the Jacobian determinant of $h$ is given by

$$
J_{h}=\frac{\partial}{\partial x}(\operatorname{Re} h(x+i y)) \cdot \frac{\partial}{\partial y}(\operatorname{Im} h(x+i y))=h_{y}(x)+h_{y}^{\prime}(x) x \quad \text { a.e. },
$$

from which we calculate that $J_{h} \geq 1$ almost everywhere in $S$. 


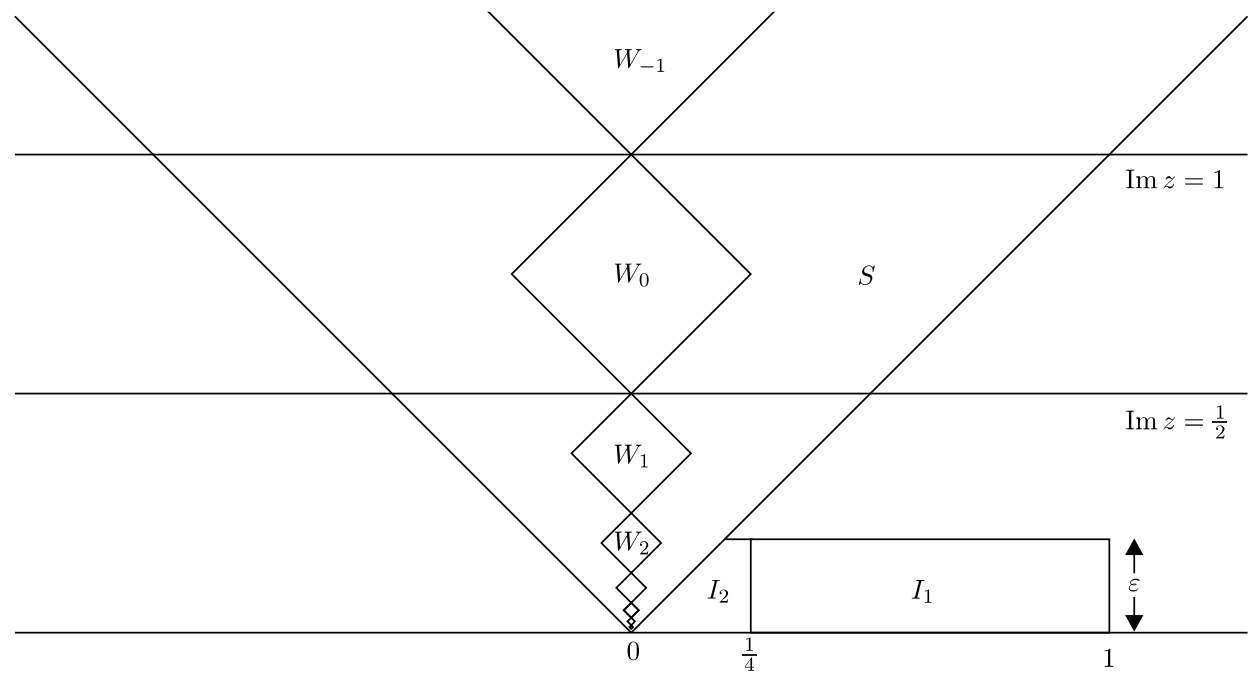

FiguRE 1. Some of the sets used in the construction.

For $x+i y \in S$ with $|x|>y$, we have that $h(x+i y)=4 x+i y$, and hence $|D h(x+i y)|=4$. By appealing to a compactness argument, there exists $M>0$ such that $|D h(x+i y)| \leq M$ for almost every $x+i y \in \bar{S}$ with $|x| \leq y$. Therefore, $h$ is $K$-quasiconformal on $S$ with $K=\max \left\{4^{2}, M^{2}\right\}$.

We now claim that if $x_{0}>0$ and $x_{0}+i y \in \bar{S} \backslash \overline{W_{0}}$, then

$$
x_{n}:=\operatorname{Re}\left(h^{n}\left(x_{0}+i y\right)\right) \rightarrow+\infty \quad \text { as } n \rightarrow \infty .
$$

That is, when $h$ is iterated, points to the right of $W_{0}$ tend to infinity through the right half of $S$. To prove the claim, note that $h_{y}\left(x_{0}\right)>1$ and that $x_{n+1}=h_{y}\left(x_{n}\right) x_{n}$ by (3.1). Using the fact that $h_{y}(x)$ is increasing for $x>0$, it follows by induction that $x_{n} \geq\left(h_{y}\left(x_{0}\right)\right)^{n} x_{0}$, which establishes (3.3).

The next step is to define, for $z=x+i y \in \mathbb{C}$,

$$
f(z)= \begin{cases}2^{-(m+1)} h\left(2^{m} z\right), & \operatorname{Im} z \in\left(2^{-(m+1)}, 2^{-m}\right], m \in \mathbb{Z}, \\ 2 x+i y / 2, & \operatorname{Im} z \leq 0 .\end{cases}
$$

Then $f$ is $K$-quasiconformal because in the upper half-plane it is locally the composition of the $K$-quasiconformal map $h$ with two (conformal) scalings. The continuity of $f$ follows from (3.2).

Let $W_{k}=2^{-k} W_{0}$ as shown in Figure 1. We note that $f(z)=z / 2$ on $\bigcup W_{k}$, since $h$ is the identity map on $W_{0}$. In fact, $\operatorname{Im} f(z)=\operatorname{Im} z / 2$ for all $z$ by (3.1). Moreover, $f(x+i y)=2 x+i y / 2$ in the region $\{x+i y:|x| \geq y\}$. These remarks could be used together with the next lemma to give a complete description of the dynamics of $f$.

Lemma 3.1. Let $z$ be such that $\operatorname{Re} z>0$ and $z \notin \bigcup \overline{W_{k}}$. Then for all sufficiently large $N$, we have

$$
\operatorname{Re}\left(f^{N}(z)\right)>\operatorname{Im}\left(f^{N}(z)\right)
$$

Proof. This is trivial unless $\operatorname{Im} z>0$, in which case we take $m \in \mathbb{Z}$ such that $\operatorname{Im} z \in\left(2^{-(m+1)}, 2^{-m}\right]$. Then $f^{N}(z)=2^{-(N+m)} h^{N}\left(2^{m} z\right)$, and so it will suffice to 
show that, for large $N$,

$$
\operatorname{Re}\left(h^{N}\left(2^{m} z\right)\right)>\operatorname{Im}\left(h^{N}\left(2^{m} z\right)\right)=\operatorname{Im}\left(2^{m} z\right) .
$$

Since $z \notin \bigcup \overline{W_{k}}$, we have that $2^{m} z \in \bar{S} \backslash \overline{W_{0}}$, and hence (3.3) gives (3.5) for all large $N$.

\subsection{A polynomial type map of high degree with wandering domains.}

The mapping $f$ has many of the dynamical properties that we require, but it is an injective function. By composing with another mapping, we now produce a quasiregular map of large degree. Choose an odd integer $d$ such that $d>2 K$. Let $\delta>0$ be small and define $p=g \circ f$, where

$$
g(z)= \begin{cases}z, & |z| \leq 1 \\ z+\delta(|z|-1) z^{d}, & 1<|z| \leq 2 \\ z+\delta z^{d}, & |z|>2\end{cases}
$$

Hence

$$
p(z)=f(z) \quad \text { whenever } \quad|f(z)| \leq 1 .
$$

As in [2, Examples 5.2-5.4], $g$ is a quasiregular map of degree $d$ and the dilatation $K(g) \rightarrow 1$ as $\delta \rightarrow 0$. We choose $\delta$ small enough that $K(g) \leq 2$. Therefore, $p$ is a quasiregular mapping of polynomial type such that

$$
K(p) \leq K(g) K(f) \leq 2 K<d=\operatorname{deg} p .
$$

Using (3.6), we note that, for $k \geq 0$,

$$
p\left(\overline{W_{k}}\right)=\overline{W_{k+1}},
$$

and so $p^{n}(z) \rightarrow 0$ on $\overline{W_{k}}$ as $n \rightarrow \infty$. Moreover, these sets $W_{k}$ lie in the Fatou set of $p$. Our aim is now to show that the $W_{k}$ are in fact components of $F(p)$ and of $\overline{I(p)}^{c}$.

We consider the escaping set $I(p)$. For all real $x>0$, we see that $f(x)=2 x$ and $p(x)=g(2 x) \geq 2 x$, and hence $x \in I(p)$. Then, since $I(p)$ is open by Theorem 1.1. there exists $\varepsilon \in\left(0, \frac{1}{4}\right)$ such that

$$
I_{1}=\left\{x+i y: \frac{1}{4} \leq x \leq 1,0 \leq y \leq \varepsilon\right\} \subseteq I(p) .
$$

Let $I_{2}=\left\{x+i y: y<x \leq \frac{1}{4}, 0 \leq y \leq \varepsilon\right\}$, as shown in Figure 1. Recalling (3.6) and the paragraph preceding Lemma 3.1, we find that if $x+i y \in I_{2}$, then $p(x+i y)=2 x+i y / 2$. Thus, each point in $I_{2}$ has some forward iterate under $p$ that lies in $I_{1}$. This implies that $I_{2} \subset I(p)$.

Now choose $j \in \mathbb{N}$ such that $2^{-j} \leq \varepsilon$ and let $w \in \partial W_{j}$ with $\operatorname{Re} w \geq 0$. Our next task is to show that $w \in \partial I(p)$. For all small $\eta>0$, the point $z=w+\eta$ satisfies the hypothesis of Lemma 3.1, and so there exists $N \in \mathbb{N}$ such that (3.4) holds but $\operatorname{Re}\left(f^{n}(z)\right) \leq \operatorname{Im}\left(f^{n}(z)\right)$ for $0 \leq n<N$. We note that

$$
0 \leq \operatorname{Im}\left(f^{n}(z)\right)=2^{-n} \operatorname{Im} z=2^{-n} \operatorname{Im} w \leq 2^{-n} \varepsilon, \quad n \geq 0 .
$$

It follows from the definition of $f$ and the above that

$$
0 \leq \operatorname{Re}\left(f^{N}(z)\right) \leq 2 \operatorname{Re}\left(f^{N-1}(z)\right) \leq 2 \operatorname{Im}\left(f^{N-1}(z)\right) \leq 2 \varepsilon .
$$

Therefore, we have that $\left|f^{n}(z)\right|<1$ for $n=0,1, \ldots, N$, and using (3.6) we deduce that $p^{n}(z)=f^{n}(z)$ for $n=0,1, \ldots, N$. By (3.4) and the above estimates, it follows that $p^{N}(z)=f^{N}(z) \in I_{1} \cup I_{2}$. This implies that $w+\eta \in I(p)$ for all small $\eta>0$, which, together with (3.7), completes the proof that $w \in \partial I(p)$. 
Since the integer $d$ in the definition of $g$ was chosen to be odd, the function $p$ is symmetric about the imaginary axis in the sense that $p(-\bar{z})=-\overline{p(z)}$. The escaping set $I(p)$ must share this symmetry, and therefore any point of $\partial W_{j} \cap\{w: \operatorname{Re} w \leq 0\}$ must also belong to $\partial I(p)$. Hence $\partial W_{j} \subseteq \partial I(p)$ and, using the complete invariance of $\partial I(p)$ under $p$, we see that in fact $\partial W_{k} \subseteq \partial I(p)$ for all $k \geq 0$. Using (3.7), it now follows that $\left(W_{k}\right)_{k \geq 0}$ are wandering components of $\overline{I(p)}^{c}$.

To show that $\left(\bar{W}_{k}\right)_{k \geq 0}$ are also components of the Fatou set $F(p)$, we need only observe that the family $\left\{p^{n}\right\}$ cannot be normal in any neighbourhood of a point of $\partial W_{k}$, as these points lie on the boundary of the escaping set.

It remains to prove the assertion made in the introduction that $J(p) \neq \partial I(p)$. To do this, let $j$ be large and consider $z_{0} \in \partial W_{j}$ not a vertex of $W_{j}$. If $U$ is a sufficiently small neighbourhood of $z_{0}$, then, by an argument similar to that used above, it can be shown that $U \backslash \overline{W_{j}} \subseteq I(p)$. Recall (3.7) and the fact that $\overline{W_{j-1}}$ does not meet $I(p)$. Thus, for example, $W_{j-1} \cap\left(\bigcup_{n} p^{n}(U)\right)=\emptyset$, and this implies that $z_{0} \notin J(p)$.

3.3. A modification with dilatation near 1. In this section, we outline how to modify the preceding construction to obtain a quasiregular map $p$ that has dilatation close to 1 , arbitrary degree $d \geq 2$, and wandering Fatou components.

To this end, let $\lambda>1$ and rescale the diamonds used earlier so that $W_{0}$ has vertices at $i$ and $i / \lambda$, and $W_{k}=\lambda^{-k} W_{0}$. For $y \in\left[\lambda^{-1}, 1\right]$, we now instead define $h_{y}(x)$ by setting $h_{y}(x)=1$ when $x+i y \in W_{0}$, setting $h_{y}(x)=\lambda^{2}$ when $|x| \geq y$ and linearly interpolating between these values. As before, we define a quasiconformal map $h$ on the strip $\left\{z: \lambda^{-1}<\operatorname{Im} z<1\right\}$ by (3.1), but now we find that the dilatation $K(h)$ tends to 1 as $\lambda \rightarrow 1$. For $z=x+i y \in \mathbb{C}$, we take

$$
f(z)= \begin{cases}\lambda^{-(m+1)} h\left(\lambda^{m} z\right), & \operatorname{Im} z \in\left(\lambda^{-(m+1)}, \lambda^{-m}\right], m \in \mathbb{Z} \\ \lambda x+i y / \lambda, & \operatorname{Im} z \leq 0\end{cases}
$$

and note that $K(f)=K(h)$. Observe that $f(z)=z / \lambda$ on $\bigcup W_{k}$ and that the dynamical behaviour of this modified function $f$ is similar to that of the original; in particular, Lemma 3.1 continues to hold.

For an arbitrary integer $d \geq 2$, we define $g(z)$ as in Section 3.2 and we again take $p=g \circ f$. Then $p$ is quasiregular with $\operatorname{deg} p=d$ and, because $K(p) \leq K(g) K(f)$, we can make the dilatation $K(p)$ arbitrarily close to 1 by choosing $\lambda$ near 1 and $\delta$ near 0 in the definition of $g$.

Proceeding as before, it is clear that $p\left(W_{k}\right)=W_{k+1}$ for $k \geq 0$ and hence that $W_{k} \subseteq F(p)$. Furthermore, it is not too difficult to see that the family $\left\{p^{n}\right\}$ is not normal near any point of $\partial W_{k}$ when $k \geq 0$, and thus $\left(W_{k}\right)_{k \geq 0}$ are wandering components of the Fatou set $F(p)$. If $d$ is chosen to be odd, then the method of Section 3.2 can be used to show that $\left(W_{k}\right)_{k \geq 0}$ are also wandering components of $\overline{I(p)}^{c}$. Note, however, that the argument used there breaks down when $d$ is even because the negative real axis is not contained in $I(p)$.

Remark. A quasiregular mapping is called uniformly quasiregular if there exists a uniform bound on the dilatation of all its iterates. By a result of Hinkkanen [6], every uniformly quasiregular mapping of the plane is quasiconformally conjugate to an entire function. We may therefore deduce from Sullivan's theorem the fact that there are no wandering domains for uniformly quasiregular mappings of the plane of polynomial type. 


\section{A 'TRANSCEndental' QUASIREgular maP WITH 'BOUNDED WANDERING'}

A long-standing open question in complex dynamics is whether there exists a transcendental entire function $g$ with a wandering component $W$ of the Fatou set, such that $\bigcup_{n} g^{n}(W)$ is a bounded set (see for example [1, Question 8] or [4, Problem 2.87]). In this section, we show that there does exist a quasiregular mapping of the plane, with an essential singularity at infinity, that has this 'bounded wandering' property.

The quasiconformal map $f$ defined in Section 3.1 has a sequence of wandering domains $W_{k}$ that converge to the origin. (Lemma 3.1 and the paragraph preceding it imply that the $W_{k}$ are wandering components of both $F(f)$ and $\overline{I(f)}^{c}$.) In fact, this remains true when $f$ is considered as a self-map of the upper half-plane. Starting from $f$, we construct a quasiregular mapping $\tilde{f}$ with the desired properties by using an interpolation technique from [3, $\S 6]$ to insert an essential singularity at infinity over the lower half-plane.

Let $\delta>0$ be small and define $\tilde{f}: \mathbb{C} \rightarrow \mathbb{C}$ by

$$
\tilde{f}(z)= \begin{cases}f(z), & \operatorname{Im} z \geq 0 \\ 2 z-\delta(\operatorname{Im} z) \exp \left(-z^{2}\right), & -1 \leq \operatorname{Im} z<0 \\ 2 z+\delta \exp \left(-z^{2}\right), & \operatorname{Im} z<-1\end{cases}
$$

It is clear that $\tilde{f}$ is continuous on $\mathbb{C}$, quasiregular on the upper half-plane and analytic on $\{z: \operatorname{Im} z<-1\}$. We show next that $\tilde{f}$ is quasiregular on

$$
D=\{z:-1<\operatorname{Im} z<0\} .
$$

Write $\phi(z)=(\operatorname{Im} z) \exp \left(-z^{2}\right)$ and let $x$ and $y$ denote the real and imaginary parts of $z$ respectively. Then $\phi$ is continuous on $D$ and the partial derivatives $\phi_{x}$ and $\phi_{y}$ are bounded on $D$ because $\exp \left(-z^{2}\right)$ tends to zero rapidly as $z \rightarrow \infty$ in $D$. If $\delta$ is chosen sufficiently small, then it follows that $\tilde{f}(z)=2 z-\delta \phi(z)$ is quasiregular on $D$; see [3, p. 647]. Here we have used the fact that the function $z \mapsto 2 z$ is analytic with derivative bounded away from zero. Therefore, $\tilde{f}$ is quasiregular on the plane.

The exponential rate of growth of $\tilde{f}(-i r) \sim \delta \exp \left(r^{2}\right)$ as real $r \rightarrow \infty$ shows that $\tilde{f}$ is not of polynomial type; see [7, Theorem 4.1] for example.

Using the fact that $\tilde{f}$ coincides with $f$ on the upper half-plane and arguing as before, we conclude that the sets $W_{k}$ (as defined in Section 3.1) are wandering components of $\overline{I(\tilde{f})}^{c}$ and of $F(\tilde{f})$. In particular, $\tilde{f}$ maps $W_{k}$ onto $W_{k+1}$, and hence in any fixed $W_{k}$ the sequence of iterates $\tilde{f}^{n}$ uniformly converges to zero as $n$ tends to infinity.

Remarks. 1. We may make the dilatation $K(\tilde{f})$ arbitrarily close to 1 by modifying the function $f$ as described in Section 3.3 and choosing $\delta>0$ to be small.

2. An important feature of the above example is that the fixed point of $\tilde{f}$ at the origin behaves like a saddle point. This type of behaviour is clearly not possible for an entire function. Moreover, Pérez-Marco [8] has proved that, for an entire function, any orbit which converges to an irrationally indifferent fixed point is eventually constant. Thus the Fatou set of an entire function cannot have a wandering domain on which the iterates converge to a fixed point. 


\section{ACKNOWLEDGMENTS}

The author would like to thank Walter Bergweiler and Alastair Fletcher for useful discussions, and the former for bringing [8] to his attention. The author also thanks Phil Rippon and Gwyneth Stallard for asking about the existence of an example of bounded wandering with an essential singularity at infinity. Finally, the author is grateful for the referee's suggestion of a discussion along the lines of that now appearing in Section 3.3 .

\section{REFERENCES}

1. W. Bergweiler, Iteration of meromorphic functions, Bull. Amer. Math. Soc. (N.S.) 29 (1993), 151-188. MR1216719 (94c:30033)

2. W. Bergweiler, Iteration of quasiregular mappings, Comput. Methods Funct. Theory 10 (2010), 455-481. MR2791320

3. W. Bergweiler, A. Fletcher, J. K. Langley and J. Meyer, The escaping set of a quasiregular mapping, Proc. Amer. Math. Soc. 137 (2009), 641-651. MR2448586 (2010f:30045)

4. D. A. Brannan and W. K. Hayman, Research problems in complex analysis, Bull. London Math. Soc. 21 (1989), 1-35. MR967787 (89m:30001)

5. A. Fletcher and D. A. Nicks, Quasiregular dynamics on the $n$-sphere, Ergodic Theory Dynam. Systems 31 (2011), 23-31. MR2755919

6. A. Hinkkanen, Uniformly quasiregular semigroups in two dimensions, Ann. Acad. Sci. Fenn. Math. 21 (1996), 205-222. MR1375517 (96m:30029)

7. P. Järvi, On the zeros and growth of quasiregular mappings, J. Anal. Math. 82 (2000), 347362. MR,1799670 (2002f:30028)

8. R. Pérez-Marco, Sur une question de Dulac et Fatou, C. R. Acad. Sci. Paris Sér. I Math. 321 (1995), 1045-1048. MR.1360570 (97c:58131)

9. S. Rickman, Quasiregular mappings, Ergebnisse der Mathematik und ihrer Grenzgebiete 26, Springer-Verlag, Berlin, 1993. MR1238941 (95g:30026)

10. D. Sullivan, Quasiconformal homeomorphisms and dynamics I. Solution of the Fatou-Julia problem on wandering domains, Ann. of Math. (2) 122 (1985), 401-418. MR819553 (87i:58103)

11. D. Sun and L. Yang, Quasirational dynamical systems (in Chinese), Chinese Ann. Math. Ser. A 20 (1999), 673-684. MR1741007 (2001d:37058)

12. D. Sun and L. Yang, Quasirational dynamic system, Chinese Science Bull. 45 (2000), 12771279 .

13. D. Sun and L. Yang, Iteration of quasi-rational mapping, Progr. Natur. Sci. (English Ed.) 11 (2001), 16-25. MR 1831577 (2002b:37059)

14. The escaping set in transcendental dynamics. Abstracts from the mini-workshop held December 6-12, 2009. Organized by Walter Bergweiler and Gwyneth M. Stallard, Oberwolfach Rep. 6 (2009), no. 4, 2927-2964. MR 2724312

School of Mathematical Sciences, University of Nottingham, Nottingham, NG7 2RD, UNITED KINGDOM

E-mail address: dan.nicks@nottingham.ac.uk 